\title{
Clinical value of IncRNA CCAT1 in serum extracellular vesicles as a potential biomarker for gastric cancer
}

\author{
KE XIAO, ZHAOGANG DONG, DING WANG, MIN LIU, JUAN DING, \\ WENDAN CHEN, ZIQI SHANG, CONGBO YUE and YI ZHANG
}

Department of Clinical Laboratory, Qilu Hospital of Shandong University, Jinan, Shandong 250012, P.R. China

Received November 29, 2020; Accepted March 3, 2021

DOI: $10.3892 / 01.2021 .12708$

\begin{abstract}
Long non-coding RNAs (lncRNAs) in extracellular vesicles (EVs) are considered to be novel non-invasive biomarkers for gastric cancer (GC). lncRNA colon cancer-associated transcript 1 (CCAT1) is aberrantly expressed in certain types of cancer. However, the role of EV lncRNA CCAT1 in patients with GC remains unclear. The current study aimed to assess the expression levels of lncRNA CCAT1 in the serum EVs of patients with GC and evaluate its potential clinical value. EVs were isolated from serum using a commercial kit and ultracentrifugation, and were identified by transmission electron microscopy, nanoparticle tracking analysis and western blotting. Serum EV lncRNA CCAT1 levels in patients with GC, chronic gastritis or atypical hyperplasia and healthy control subjects were detected by reverse transcription-quantitative PCR. Additionally, lncRNA CCAT1 was detected in GC and adjacent non-cancerous tissue samples. Serum EVs were successfully isolated and identified in all patients. The results revealed that serum EV lncRNA CCAT1 levels in patients with GC were significantly higher compared with those in healthy controls, patients with chronic gastritis or atypical hyperplasia (all $\mathrm{P}<0.05$ ). Additionally, EV IncRNA CCAT1 expression levels were significantly different among various groups based on the depth of invasion, distant metastasis and the Tumor-Node-Metastasis stage. The area under the curve (AUC) value of EV lncRNA CCAT1 was 0.890 [95\% confidence interval (CI), 0.826-0.937] with $79.6 \%$ sensitivity and $92.6 \%$ specificity. The combination of EV
\end{abstract}

Correspondence to: Professor Yi Zhang, Department of Clinical Laboratory, Qilu Hospital of Shandong University, 107 Wenhuaxi Road, Jinan, Shandong 250012, P.R. China

E-mail: yizhang@sdu.edu.cn

Abbreviations: lncRNA, long non-coding RNA; EVs, extracellular vesicles; GC, gastric cancer; CCAT1, colon cancer-associated transcript 1; OS, overall survival; DFS, disease-free survival; CEA, carcinoembryonic antigen; CA19-9, carbohydrate antigen 19-9; CA72-4, carbohydrate antigen 72-4

Key words: gastric cancer, colon cancer-associated transcript 1, extracellular vesicle, long non-coding RNA, biomarker
lncRNA CCAT1 and carcinoembryonic antibody produced an AUC value of 0.910 (95\% CI, 0.849-0.951) with the sensitivity and specificity of 80.5 and $92.6 \%$, respectively. In addition, lncRNA CCAT1 was determined to be stable in serum EVs. The expression levels of lncRNA CCAT1 in GC tissue were positively correlated with those in serum EVs, and high levels of lncRNA CCAT1 were associated with a low disease-free survival rate in patients with GC. The results of the present study demonstrated that serum EV lncRNA CCAT1 levels were upregulated in patients with GC compared with those healthy subjects and patients with other illnesses, and may therefore be used as a novel biomarker for this type of cancer.

\section{Introduction}

Gastric cancer (GC) is the third leading cause of cancer-associated deaths worldwide (1). The 5-year survival rate of patients with advanced GC is only $5-20 \%$, with a median overall survival of 10 months worldwide $(2,3)$. Therefore, early detection of GC is crucial for improving patient survival rates (4). Currently, endoscopy and pathological examination are the diagnostic gold standard; however, these procedures are invasive and costly. The detection of biomarkers in peripheral blood is a useful method for GC screening. The traditional tumor markers used for GC detection include carcinoembryonic antigen (CEA), carbohydrate antigen 19-9 (CA19-9) and carbohydrate antigen 72-4 (CA72-4) (5). However, previous studies by our group (5) and Wang et al (6) have demonstrated that the sensitivity and specificity of the three traditional markers are insufficient for the diagnosis of GC, which limits their clinical utility. Thus, the identification of a diagnostic marker with high sensitivity and specificity for patients with GC is urgently required.

In the past decade, there has been notable progress in the field of study of extracellular vesicles (EVs), which have attracted increasing attention due to their widespread prevalence in various body fluids $(7,8)$, ease of access $(9,10)$ and association with various diseases (11). EVs are a heterogeneous population of cell-derived vesicles secreted by various cell types to mediate their intracellular communication (12). Following uptake by recipient cells, EVs serve a certain role in cancer progression; for example, Abd Elmageed et al (13) have reported that microRNA (miRNA or miR)-125b, miR-130 and miR-155, as well as HRas and Kras mRNAs in EVs from 
prostate cancer cells participate in neoplastic reprogramming and tumor formation of adipose stem cells. This hypothesis also has been supported by Zhang et al (14), who demonstrated that EVs derived from GC cells induce neutrophils to polarize to N2 tumor-associated neutrophils, thus promoting GC cell migration. EV membranes are enriched in various protein markers including CD9, CD63 and tumor susceptibility gene 101 (TSG101) (5). By means of EVs, various molecules such as lipids, proteins and nucleic acids [(DNA, mRNA and long non-coding RNA (lncRNA)] can be transferred from donor to recipient cells (15), suggesting that this mechanism may be involved in GC progression. In addition, circulating EVs are present in liquid biopsies and may be used as diagnostic markers of GC $(16,17)$; therefore, EVs may have potential diagnostic value in $\mathrm{GC}$.

IncRNAs have been reported to serve crucial roles in cancer development and progression (18). IncRNAs have a number of functions in various biological processes, including the cis or trans transcriptional and post-transcriptional regulation of RNA (19). An increasing number of lncRNAs have been demonstrated to participate in GC progression. For example, knockdown of lncRNA AF147447 promotes GC cell proliferation and invasion by directly binding to miR-34c (20). Gastric cancer metastasis-associated lncRNA (GMAN) promotes the expression of EphrinA1 through the GMAN-antisense pathway, promoting GC metastasis (21). In addition, circulating EVs loaded with specific bioactive molecules reflect the status of GC progression and metastasis (4). The EV-mediated transfer of lncRNA HOX transcript antisense RNA (HOTAIR) promotes cisplatin resistance in liver cancer cells by regulating the high mobility group AT-hook (HMGA)1/miR-218 axis (22). lncRNAs in EVs are stably present in circulation and are regarded as useful markers that are reflective of disease status. Previous studies have reported that certain EV lncRNAs are highly expressed in the serum of patients with GC, including LINC00152, IncRNA HOTTIP and lncUEGC1 (23-25). The results of the aforementioned studies suggest that EV lncRNAs may be used as auxiliary biomarkers of GC. However, certain limitations, such as their lack of clinical efficiency and clinical effect require further consideration.

IncRNA colon cancer-associated transcript-1 (CCAT1) is an oncogenic lncRNA located on chromosome 8q24, close to the gene encoding c-Myc (26). IncRNA CCAT1 has been reported to be elevated in certain types of cancer, such as GC (27), colorectal cancer (CRC) (28) and hepatocellular carcinoma (29). Additionally, lncRNA CCAT1 serves a crucial role in various biological processes, such as cell proliferation and invasion (30). Aberrant expression of 1ncRNA CCAT1 affects tumorigenesis, tumor size, lymph node metastasis, Tumor-Node-Metastasis (TNM) stage, tumor differentiation and invasion $(31,32)$. A previous study by Shen et al (33) has revealed that IncRNA CCAT1 serves a role in cervical cancer by promoting cell proliferation and invasion via the regulation of the miR-181a-5p/MMP14 axis. You et al (34) have also demonstrated that CCAT1 promotes the proliferation of prostate cancer cells by facilitating androgen receptor (AR) expression as a scaffold for DEAD-box helicase 5 (P68) and the AR transcriptional complex, and by acting as a sponge of miR-28-5p to prevent its anticancer effects. IncRNA CCAT1 can be used as a diagnostic marker; Abedini et al (35) have demonstrated that the levels of plasma lncRNA CCAT1 in patients with $\mathrm{CRC}$ are higher compared with those in healthy control subjects. In GC, IncRNA CCAT1 contributes to tumor growth and invasion by targeting miR-219-1 (36). This suggests that lncRNACCAT1 is involved in the progression of GC and may serve as a potential biomarker.

IncRNAs can exist stably in the peripheral circulation and are protected by EVs. As markers, EVs have a good diagnostic value in GC; for example, a previous study has demonstrated that lncRNA zinc finger NFX1-type containing 1 antisense RNA 1 levels are elevated in the serum exosomes of patients with GC compared with those in healthy controls (37). Furthermore, Li et al (23) have determined that plasma LINC00152 levels are significantly higher in patients with GC compared with those in healthy controls. IncRNA CCAT1 has also been identified in the plasma and serum EVs of patients with CRC $(38,39)$. However, to the best of our knowledge, there no studies are currently available on EV lncRNA CCAT1 in patients with GC. Therefore, the current study aimed to identify the levels of IncRNA CCAT1 in the serum EVs of patients with GC and evaluate its expression and diagnostic value. In addition, the correlation of IncRNA CCAT1 with various clinicopathological variables was assessed. The present study further aimed to identify a reliable biomarker in the diagnosis of GC.

\section{Materials and methods}

Patients and control subjects. Serum samples of 27 healthy control subjects, 26 patients with chronic gastritis, 30 patients with atypical hyperplasia and 113 patients with GC were collected at the Qilu Hospital of Shandong University between January and December 2017. Peripheral blood samples from the GC group were collected before surgery. The age ranges in the subject groups were as follows: Healthy controls, 29-62 (male subjects, $\mathrm{n}=8$ ) and 30-66 (female subjects, $\mathrm{n}=19$ ); chronic gastritis, 32-69 (male patients, $n=14$ ) and 33-65 (female patients, $\mathrm{n}=12$ ); atypical hyperplasia 33-73 (male patients, $n=21$ ) and 41-73 (female patients, $n=9$ ); GC, 25-82 (male patients, $n=84$ ) and 23-74 (female patients, $n=29$ ) years. In addition, 16 paired $\mathrm{GC}$ and adjacent non-cancerous tissues were collected during surgical resection and stored at $-80^{\circ} \mathrm{C}$ immediately. The distance between the tumor margin and the adjacent non-tumor tissue $\geq 5 \mathrm{~cm}$. The demographic and clinicopathological characteristics of all participants were recorded. GC was diagnosed by two pathologists and staged according to the Union for International Cancer Control/American Joint Committee on Cancer TNM staging system (8th edition) (40). No patients had received any adjuvant therapy such as radiotherapy or chemotherapy prior to sample collection. The inclusion criteria for the healthy control subjects were routine blood test, biochemical index, tumor marker and imaging test results determined to be within the reference range during physical examination. Outliers were defined through Tukey's box-plot method (41), and 4, 5, 3 and 6 suspected outliers were removed from healthy controls, patients with chronic gastritis, atypical hyperplasia and GC, respectively. In addition, four pairs of GC and adjacent non-cancerous tissues were removed as outliers. The present study was approved by the Medical Ethics Committee of Qilu Hospital of Shandong University 
(approval no. KYLL-2015-097). All participants provided signed informed consent.

Isolation of EVs from serum. Serum was collected from $5 \mathrm{ml}$ venous blood by centrifuging at 3,000 $\mathrm{x}$ g for $10 \mathrm{~min}$ at room temperature. The samples were filtered through a $0.22-\mu \mathrm{m}$ filter (Millipore Sigma) to eliminate cell fragments and organelles. Two methods were used to isolate EVs. For the commercial kit method, the serum was centrifuged at 50,000 $\mathrm{x}$ g for $60 \mathrm{~min}$ to remove large microbubbles, and the subsequent procedures were performed according to the manufacturer's instructions of the exoEasyMaxi kit (cat. no. 76064; Qiagen GmbH). Briefly, $1 \mathrm{ml}$ serum was mixed with $1 \mathrm{ml} \mathrm{XBP}$ buffer and added to the exoEasy spin column (in a 50-ml collection tube to bind EVs). Following centrifugation at $500 \mathrm{x}$ g for $60 \mathrm{sec}, 10 \mathrm{ml} \mathrm{XWP}$ buffer was added and centrifuged at 5,000 $\mathrm{x} \mathrm{g}$ for $5 \mathrm{~min}$, and $400 \mu 1 \mathrm{XE}$ buffer was used to elute EVs at $500 \mathrm{x}$ g for $5 \mathrm{~min}$. For ultracentrifugation, $1 \mathrm{ml}$ serum was diluted 1:7 in PBS and centrifuged at $120,000 \mathrm{x}$ g for $2 \mathrm{~h}$. A lamellar precipitate was observed at the bottom of the tube, the main component of which was EVs. The supernatant was discarded and the EVs were resuspended with $100 \mu$ l PBS. All centrifugation steps were performed at $4^{\circ} \mathrm{C}$ unless stated otherwise.

Transmission electron microscopy (TEM). A total of $10 \mu 1$ EV suspension was added to a 200-mesh carbon film copper net. The carbon film was allowed to absorb EVs for $20 \mathrm{~min}$ in a dry environment. The copper net was sequentially placed in $50 \mu \mathrm{l} 11 \%$ glutaraldehyde, $100 \mu \mathrm{l} \mathrm{dd}_{2} \mathrm{O}, 50 \mu \mathrm{l}$ uranyl acetate and $50 \mu 1$ methylcellulose-uranyl acetate droplets. The excess liquid was gently removed using filter paper, leaving a thin methylcellulose film, which was dried for 5-10 min at room temperature. The samples were observed in 5-10 fields per sample under a Hitachi HT7800 transmission electron microscope (Hitachi High-Tech Corporation) at $80 \mathrm{kV}$ under x40,000 magnification.

Measurement of size distribution. The size distribution of EVs was observed on a ZetaView analyzer (Particle Metrix GmbH) according to the manufacturer's instructions. Nanoparticle Tracking Analysis (NTA) was used to capture the Brownian motion of each particle in the video. Based on the different diffusion movements of large and small particles in the surrounding liquid, the hydrodynamic diameters of particles were determined. In addition, the charge state of particle surface (zeta potential) was measured by determining the movement of particles in an applied electric field.

EV protein quantification and western blot analysis. EVs were suspended in RIPA lysis buffer (cat. no. P0013B; Beyotime Institute of Biotechnology) on ice to dissolve the protein. Total protein concentration was determined by BCA protein detection kit (cat. no. P0012; Beyotime Institute of Biotechnology). The extracted protein was diluted with loading buffer 4:1 and incubated at $100^{\circ} \mathrm{C}$ for $5 \mathrm{~min}$. A total of $\sim 20 \mu \mathrm{g} \mathrm{EV}$ protein per lane was separated by $10 \%$ sodium dodecyl sulfate polyacrylamide gel electrophoresis, transferred to PVDF membranes, blocked in 5\% non-fat milk for $1 \mathrm{~h}$ and incubated overnight at $4^{\circ} \mathrm{C}$ with primary mouse anti-CD63 (cat. no. ab59479; 1:1,000; Abcam), rabbit anti-TSG101 (cat. no. ab125011;
1:1,000; Abcam), rabbit anti-CD9 (cat. no. 13403s; 1:1,000; Cell Signaling Technology, Inc.), rabbit anti-GAPDH (cat. no. AB-P-R 001; 1:1,000; Hangzhou Good Here Biotechnology Co., Ltd.) and rabbit anti-calnexin (cat. no. 2679s; 1:1,000; Cell Signaling Technology, Inc.) antibodies. The washing reagent TBST (cat. no. T1081; Beijing Solarbio Science \& Technology Co., Ltd.) used to remove the primary antibodies The horseradish peroxidase-labeled goat anti mouse (cat. no. A0216; 1:2,000; Beyotime Institute of Biotechnology) or goat anti rabbit antibody (cat. no. A0208; 1:2,000; Beyotime Institute of Biotechnology) was used to imprint the target protein and incubated at room temperature for $1 \mathrm{~h}$. The diluent for the secondary antibody (cat. no. WB100D) was purchased from New Cell \& Molecular Biotech Co., Ltd. The protein signals were detected by an enhanced chemiluminescence detection system (Amersham Imager 680; Cytiva).

$R N A$ extraction and reverse transcription-quantitative ( $R T-q)$ $P C R$. Serum and tissue RNAs were extracted using an exoRNeasy Serum/Plasma Midi kit (cat. no. 77044; Qiagen GmbH) and RNAprep pure FFPE kit [cat. no. DP439; TIANGEN Biotech (Beijing) Co., Ltd.] according to the manufacturer's instructions. An All-in-one ${ }^{\mathrm{TM}}$ First-Strand cDNA Synthesis kit (cat. no. QP006; GeneCopoeia, Inc.) was used to reverse-transcribe an equal amount of total RNA from each sample to cDNA according to the manufacturer's instructions. cDNA was diluted 1:5 with $\mathrm{ddH}_{2} \mathrm{O}$, and qPCR was performed using a CFX96 qPCR instrument (Bio-Rad Laboratories, Inc.) with an All-in-one ${ }^{\mathrm{TM}}$ qPCR Mix (cat. no. QP001; GeneCopoeia, Inc.). The primers used were IncRNA CCAT1 forward, 5'-AGGGGCTCTCCTTTTGTTCG-3' and reverse, 5'-GATTTCAGCAGCGAGGGTCT-3' (88 bp); GAPDH (cat. no. B662104; 138 bp) was purchased from Sangon Biotech Co., Ltd. The thermocycling conditions were as follows: Initial denaturation at $95^{\circ} \mathrm{C}$ for $10 \mathrm{~min}$, followed by 40 cycles of $95^{\circ} \mathrm{C}$ for $15 \mathrm{sec}, 62^{\circ} \mathrm{C}$ for $20 \mathrm{sec}$ and $72^{\circ} \mathrm{C}$ for $10 \mathrm{sec}$. The amplification specificity was determined by melting curve analysis. Data were normalized to GAPDH, and relative expression levels were evaluated using the $2^{-\Delta \Delta \mathrm{Cq}}$ method (42). No template reaction and no RT reaction were used as negative controls.

Stability of serum EV lncRNA CCAT1 in harsh environments. EVs from patient serum were divided into three groups that were untreated (control), treated with RNase A or treated with RNase A plus $0.1 \%$ Triton X-100 for $45 \mathrm{~min}$ at $37^{\circ} \mathrm{C}$, respectively. In order to evaluate the stability of IncRNA CCAT1 in serum EVs in harsh environments, serum samples were stored at room temperature or $4^{\circ} \mathrm{C}$ for 24 or $48 \mathrm{~h}$. In addition, serum samples were frozen and thawed three times at $-80^{\circ} \mathrm{C}$, and fresh serum used as a control. Following these treatments, the expression levels of IncRNA CCAT1 were detected by RT-qPCR.

Fecal occult blood tests. The bleeding of digestive tract was measured by colloidal gold-based Fecal Occult Blood diagnostic kit (Shanghai Chemtron Biotech Co., Ltd.).

Online bioinformatics analysis. The expression levels of EV lncRNA CCAT1 were assessed in various diseases using the online database exoRBase (http://www.exorbase.org/), which is a repository of IncRNAs derived from RNA-seq data analyses of 


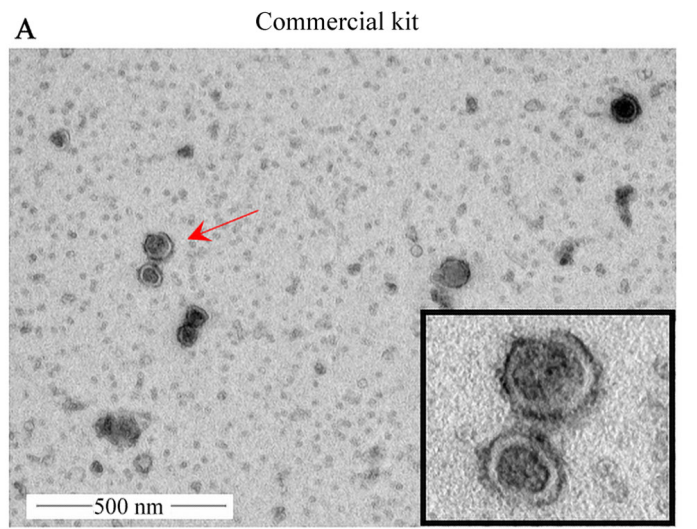

B
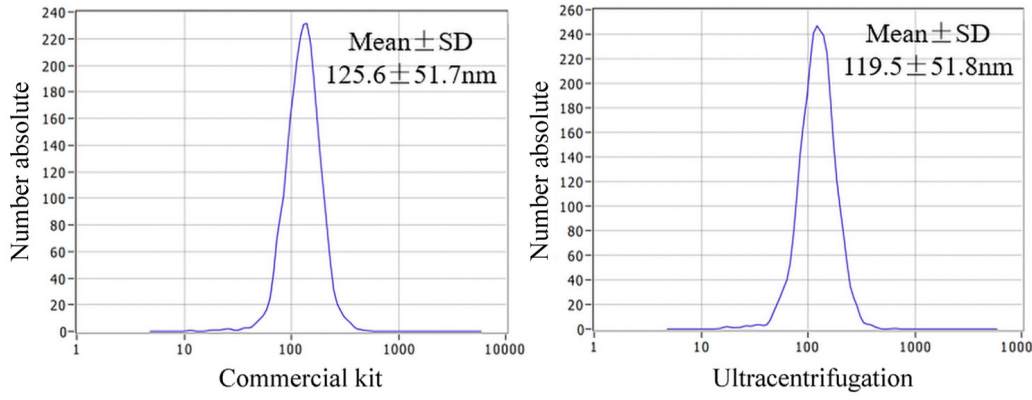

Ultracentrifugation

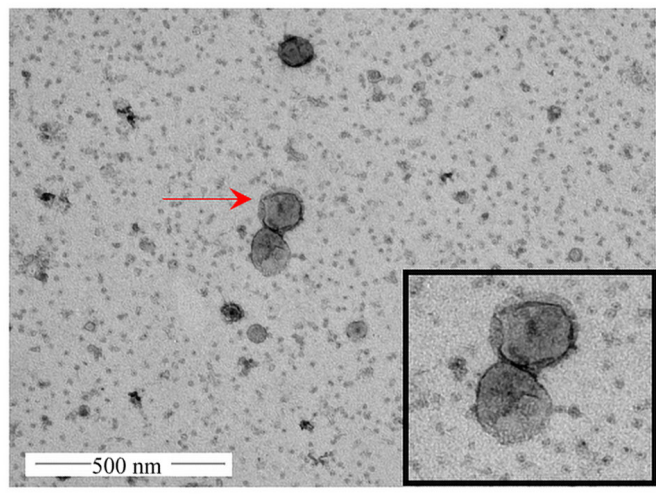

$\mathrm{C}$

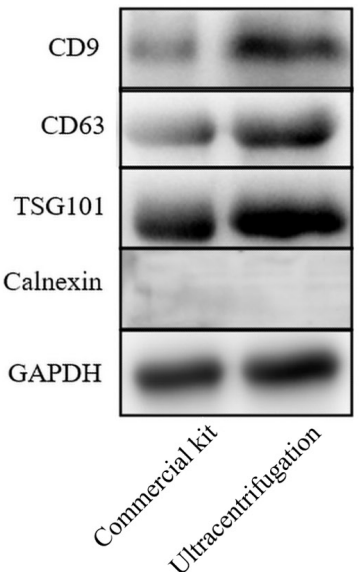

Figure 1. Characterization of serum EVs. EVs were isolated and characterized from human serum using a commercial kit and ultracentrifugation. (A) Transmission electron microscopy micrographs. Magnification, x40,000. (B) The size distribution of EVs was determined using a particle tracking analyzer. (C) Western blot analysis of EV markers CD9, CD63, TSG101 and calnexin. EVs, extracellular vesicles; TSG101, tumor susceptibility gene 101.

human blood exosomes (43). GEPIA (http://gepia.cancer-pku. cn/index.html) was used to obtain the Kaplan-Meier curve to analyze the association between the expression of CCAT1 and survival times (months) of patients, including overall survival (OS) and disease-free survival (DFS).

Statistical analysis. Data are presented as the median and interquartile ranges or the mean \pm SD. SPSS version 25.0 (IBM Corp.) and MedCalc version 8.0 (MedCalc Software, Ltd.) were used for statistical analyses. The Kolmogorov-Smirnov test was used to determine the data distribution in each group. Serum EV lncRNA CCAT1 levels were evaluated by the Mann Whitney U or Kruskal Wallis test in different groups based on patient clinicopathological characteristics. Data on the stability of serum EV lncRNA CCAT1 were analyzed using one-way ANOVA followed by Tukey's post hoc test or two-way ANOVA followed by Bonferroni post hoc test. IncRNA CCAT1 levels in paired samples of GC tissues were analyzed with Wilcoxon's signed rank test. Receiver operating characteristic (ROC) curves were used to evaluate the diagnostic value of biomarkers in GC, and area under the curve (AUC) was calculated. The cut-off value was calculated using the Jordan index (sensitivity + specificity-1). OS and DFS were analyzed by Kaplan-Meier estimates and examined by log-rank test. The correlations between EV lncRNA CCAT1 in the serum of patients with GC and CCAT1 in GC tissues were determined by Spearman's correlation analysis. $\mathrm{P}<0.05$ was considered to indicate a statistically significant difference.

\section{Results}

Characterization of serum EVs. EVs were extracted using a commercial kit and ultracentrifugation. The results of TEM analysis demonstrated that EVs presented with a unique morphology with dish-like vesicles and a double-lipid layer (Fig. 1A). In addition, NTA revealed that the diameters of EVs extracted using a commercial kit and ultracentrifugation were $125.6 \pm 51.7$ and $119.5 \pm 51.8 \mathrm{~nm}$, respectively (Fig. 1B). The zeta potentials for the commercial kit and ultracentrifugation were-21.41 \pm 1.93 and $-16.46 \pm 1.90 \mathrm{mV}(\mathrm{P}=0.061)$, respectively (data not shown). Surface marker proteins including CD9, CD63, TSG101, the negative control protein calnexin and GAPDH (used as a loading control) were identified in the two groups of EVs (Fig. 1C). The EVs obtained using the commercial kit were similar to those obtained by ultracentrifugation.

Serum EV lncRNA CCAT1 levels in patients with GC, chronic gastritis and atypical hyperplasia as well as healthy control subjects. The results of the bioinformatics analysis revealed that the levels of 1ncRNA CCAT1 were upregulated in hepatocellular carcinoma and pancreatic adenocarcinoma compared with those in healthy controls (Fig. S1). However, there no 


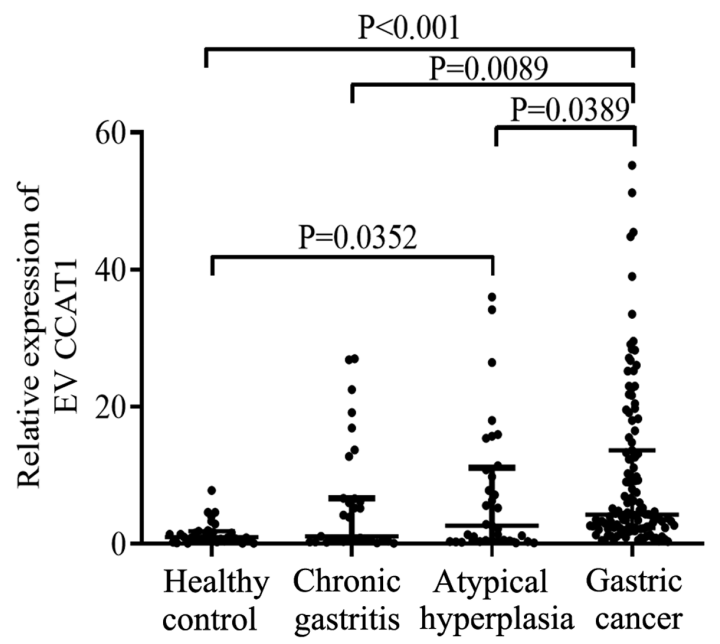

Figure 2. Serum levels of EV long non-coding RNA CCAT1 in patients with gastric cancer, atypical hyperplasia and chronic gastritis, as well as healthy control subjects. EV, extracellular vesicle; CCAT1, colon cancer-associated transcript 1 .

data were available for lncRNA CCAT1 expression in patients with GC. The data obtained in the current study revealed that serum EV lncRNA CCAT1 levels in patients with GC [4.170 (2.250-11.740)] were significantly higher compared with those in the healthy controls [0.910 (0.400-1.630)], patients with chronic gastritis $[0.710(0.406-5.453)]$ and atypical hyperplasia [1.812 (0.434-8.321)] (all $\mathrm{P}<0.05)$. Furthermore, lncRNA CCAT1 expression levels were higher in patients with atypical hyperplasia compared with those in healthy controls $(\mathrm{P}<0.05$; Fig. 2). No differences were observed in the CCAT1 expression levels between patients with chronic gastritis and healthy controls or patients with atypical hyperplasia.

Correlation between serum EV IncRNA CCAT1 levels and the clinicopathological features of patients with GC. The current study evaluated the clinical value of CEA and EV lncRNA CCAT1 in patients with GC. The results demonstrated that high expression levels of EV lncRNA CCAT1 were significantly associated with the depth of invasion $(\mathrm{P}=0.040)$, distant metastasis $(\mathrm{P}<0.001)$ and TNM stage $(\mathrm{P}<0.001)$. However, there was no significant association between EV lncRNA CCAT1 levels and patient sex, age, cancer location, Bormann classification, tumor diameter, differentiation, lymphatic metastasis, hypertension or fecal occult blood (Table I). CEA levels were associated with tumor diameter, depth of invasion and TNM stage (5).

Diagnostic value of serum EV lncRNA CCAT1 levels in GC. Serum EV lncRNA CCAT1 levels were significantly higher in patients with stage III+IV GC [7.250 (2.525-19.630)] compared with those in patients with stage I+II GC [3.490 (1.955-6.185)] $(\mathrm{P}=0.012$; Fig. 3A). This suggested that EV lncRNA CCAT1 may be a reliable biomarker of GC. The predictive value of EV lncRNA CCAT1 was subsequently determined. The AUC of EV lncRNA CCAT1 was [0.890; 95\% confidence interval (CI), 0.826-0.937)], which indicated a good capability to diagnose patients with GC. The cut-off, sensitivity and specificity values of EV lncRNA CCAT1 were 1.990, 79.6 and $92.6 \%$, respectively (Table II). IncRNA CCAT1 and CEA were combined to assess their ability to diagnose GC. ROC analysis demonstrated that the AUC of the combined markers was 0.910 (95\% CI, 0.849-0.951; Fig. 3B). The sensitivity and specificity of combined lncRNA CCAT1 and CEA were 80.5 and $92.6 \%$, respectively.

Stability of serum EV lncRNA CCAT1 in harsh environments. To determine the stability of serum EV lncRNA CCAT1, EVs from patient serum were divided into three groups. The results revealed that there was no significant differences between the RNase A treatment and control groups; however, when TritonX-100 penetrated the EVs, the expression levels of EV lncRNA CCAT1 decreased significantly (Fig. S2). These results confirmed that IncRNA CCAT1 was present in serum EVs. To verify the stability of 1ncRNA CCAT1 in serum EVs in harsh environments, serum samples were stored at room temperature or $4^{\circ} \mathrm{C}$ for 24 or $48 \mathrm{~h}$ (Fig. S3A). With fresh serum as a control, serum samples were frozen and thawed three times at $-80^{\circ} \mathrm{C}$ (Fig. S3B). The results demonstrated that the aforementioned treatments had no significant effects on the expression of IncRNA CCAT1 in EVs, which confirmed that the expression levels of serum EV lncRNA CCAT1 were stable.

Expression of IncRNA CCAT1 in GC tissues. A previous study has suggested that circulating EV IncRNA may be secreted from GC tissues (44). Therefore, the current study assessed the source of serum EV lncRNA CCAT1. The results demonstrated that lncRNA CCAT1 levels in GC tissues were significantly higher compared with those in the adjacent tissues $(\mathrm{P}<0.001$; Fig. S4A). The correlation analysis between lncRNA CCAT1 in $\mathrm{GC}$ tissues and serum EVs revealed a positive correlation $(\mathrm{r}=0.685 ; \mathrm{P}=0.003$; Fig. $\mathrm{S} 4 \mathrm{~B})$. These results suggested that serum EV lncRNA CCAT1 may be secreted from GC cells.

Prognostic value of lncRNA CCATl expression in GC. To evaluate the value of lncRNA CCAT1 in GC prognosis, GEPIA (http:/gepia.cancer-pku.cn/index.html) was used. As presented in Fig. 4A, low expression levels of lncRNA CCAT1 were significantly associated with a favorable DFS outcome compared with high expression levels of lncRNA CCAT1 in patients with $\mathrm{GC}$ [hazard ratio $(\mathrm{HR})=1.7$; log-rank $\mathrm{P}=0.038$ ]. However, it exhibited no significance in predicting OS ( $\mathrm{HR}=1.3$; log-rank $\mathrm{P}=0.310$; Fig. 4B). These results suggested that lncRNA CCAT1 may be a useful biomarker for predicting the DFS of patients with GC.

\section{Discussion}

Recent studies have demonstrated that EV lncRNAs are reliable biomarkers for various types of cancer including breast cancer, CRC and hepatocellular carcinoma (45-47). Previous studies have reported that lncRNA LINC00152, HOTTIP and lncUEGC1 in EVs may be suitable biomarkers for GC, and their AUC values were 0.657, 0.827 and 0.876, respectively (23-25). Furthermore, the sensitivity and specificity of the aforementioned three lncRNAs were 48.1 and 85.2, 69.8 and 85.0 , and 88.24 and $83.33 \%$, respectively (23-25). Notably, the AUC, sensitivity and specificity of lncRNA CCAT1 in 
Table I. Extracellular vesicle long non-coding RNA CCAT1 expression levels and patient clinicopathological characteristics.

\begin{tabular}{|c|c|c|c|c|}
\hline Variable & $\mathrm{n}$ & $\begin{array}{c}\text { CCAT1, median } \\
\text { (interquartile range) }\end{array}$ & $\mathrm{U}$ value & P-value \\
\hline Sex & & & $1,171.500$ & 0.760 \\
\hline Male & 84 & $4.200(2.285,10.910)$ & & \\
\hline Female & 29 & $4.070(2.200,13.505)$ & & \\
\hline Age, years & & & $1,315.500$ & 0.111 \\
\hline$\leq 61$ & 59 & $3.560(2.200,9.080)$ & & \\
\hline$>61$ & 54 & $4.465(2.493,16.155)$ & & \\
\hline Cancer location & & & $5.625^{\mathrm{a}}$ & 0.131 \\
\hline Antrum & 44 & $3.405(2.026,7.400)$ & & \\
\hline Angulus & 21 & $3.690(1.300,10.190)$ & & \\
\hline Cardia & 27 & $6.020(2.610,21.690)$ & & \\
\hline Body & 21 & $4.280(2.740,16.180)$ & & \\
\hline Tumor diameter & & & $1,352.000$ & 0.161 \\
\hline$\leq 3 \mathrm{~cm}$ & 56 & $3.625(2.255,6.603)$ & & \\
\hline$>3 \mathrm{~cm}$ & 57 & $4.370(2.235,15.185)$ & & \\
\hline Differentiation & & & $3.821^{\mathrm{a}}$ & 0.148 \\
\hline Well differentiated & 12 & $3.565(1.678,4.583)$ & & \\
\hline Moderately differentiated & 25 & $2.700(1.715,11.090)$ & & \\
\hline Poorly differentiated & 76 & $4.715(2.345,14.553)$ & & \\
\hline Bormann type & & & $0.180^{\mathrm{a}}$ & 0.981 \\
\hline I & 18 & $4.085(2.330,7.472)$ & & \\
\hline II & 55 & $4.230(2.200,14.840)$ & & \\
\hline III & 25 & $4.340(2.420,12.780)$ & & \\
\hline IV & 15 & $3.580(2.110,12.670)$ & & \\
\hline Invasion depth & & & $8.307^{\mathrm{a}}$ & 0.040 \\
\hline $\mathrm{T} 1$ & 26 & $3.525(2.350,5.618)$ & & \\
\hline $\mathrm{T} 2$ & 21 & $3.040(1.210,4.715)$ & & \\
\hline $\mathrm{T} 3$ & 22 & $6.650(2.790,17.353)$ & & \\
\hline $\mathrm{T} 4$ & 44 & $4.705(2.268,17.405)$ & & \\
\hline Lymphatic metastasis & & & $1,252.500$ & 0.074 \\
\hline No & 48 & $3.445(2.028,6.525)$ & & \\
\hline Yes & 65 & $4.470(2.400,15.185)$ & & \\
\hline Distant metastasis & & & 289 & $<0.001$ \\
\hline No & 95 & $3.560(2.110,6.690)$ & & \\
\hline Yes & 18 & $20.135(8.815,25.645)$ & & \\
\hline Tumor-Node-Metastasis stage & & & $21.851^{\mathrm{a}}$ & $<0.001$ \\
\hline I & 37 & $3.140(1.685,4.995)$ & & \\
\hline II & 28 & $3.935(2.038,11.840)$ & & \\
\hline III & 30 & $3.905(2.178,9.135)$ & & \\
\hline IV & 18 & $20.135(8.815,25.645)$ & & \\
\hline Hypertension & & & $1,018.500$ & 0.190 \\
\hline No & 84 & $3.775(2.208,9.228)$ & & \\
\hline Yes & 29 & $6.020(2.360,18.150)$ & & \\
\hline Fecal occult blood & & & $1,017.500$ & 0.076 \\
\hline No & 81 & $4.340(2.455,13.260)$ & & \\
\hline Yes & 32 & $3.090(1.978,7.328)$ & & \\
\hline
\end{tabular}

${ }^{\text {aH}} \mathrm{H}$-value. CCAT1, colon cancer-associated transcript 1.

the current study were $0.890,79.6$ and $92.6 \%$, respectively, which were higher compared with those of the aforementioned three lncRNAs, suggesting that it may be a reliable potential biomarker. Furthermore, the levels of EV lncRNA CCAT1 
Table II. Predictive value of CEA and long non-coding RNA CCAT1 in extracellular vesicles in gastric cancer.

\begin{tabular}{lccrr}
\hline Marker & Cut-off & Sens $(95 \%$ CI $)$ & Spec $(95 \%$ CI $)$ & AUC (95\% CI) \\
\hline CCAT1 & 1.990 & $79.600(71.000-86.600)$ & $92.600(75.700-98.900)$ & $0.890(0.826-0.937)$ \\
CCAT1 + CEA & & $80.500(72.000-87.400)$ & $92.600(75.700-98.900)$ & $0.910(0.849-0.951)$
\end{tabular}

CCAT1, colon cancer-associated transcript 1; CEA, carcinoembryonic antibody; Sens, sensitivity; Spec, Specificity; CI, confidence interval; AUC, area under the curve.
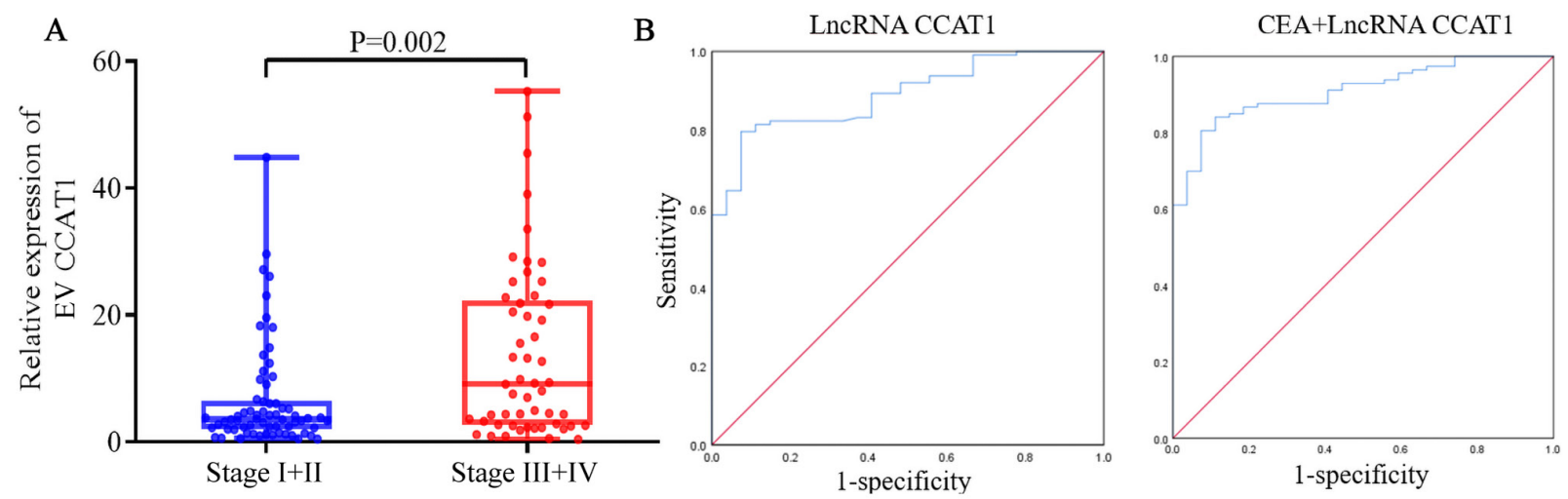

Figure 3. Diagnostic value of serum EV lncRNA CCAT1 in GC. (A) Expression of lncRNA CCAT1 in patients with GC and different Tumor-Node-Metastasis stages. IncRNA CCAT1 was normalized to GAPDH. (B) ROC curve analyzing the diagnostic value of EV lncRNA CCAT1 and CEA. EV, extracellular vesicle; lncRNA, long non-coding RNA; CCAT1, colon cancer-associated transcript 1.
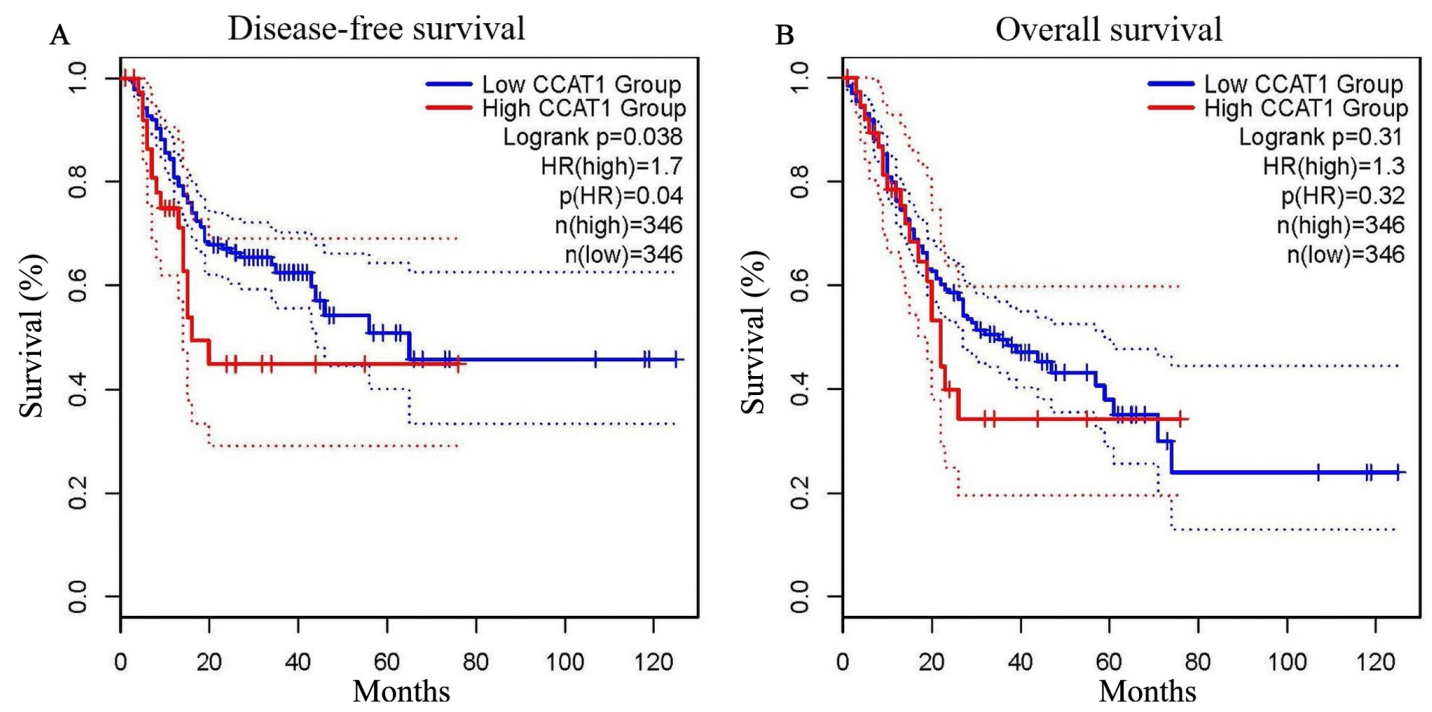

Figure 4. Prognostic value of CCAT1 in patients with gastric cancer. (A) Long non-coding RNA CCAT1 expression levels were significantly associated with disease-free survival. (B) No significant association was observed between CCAT1 expression levels and overall survival. CCAT1, colon cancer-associated transcript 1.

were associated with depth of invasion, distal metastasis and TNM stage, but not associated with other clinicopathological features, such as patient age and sex in the present study. Serum EV lncRNA CCAT1 was also positively correlated with its expression in GC tissues and associated with the prognosis of patients with GC. These results suggested that EV lncRNA CCAT1 may be a reliable tumor marker for GC.

EVs are involved in the key steps of cancer progression and have numerous potential applications in diagnosis and treatment. A previous study has demonstrated that tumor cells secrete EVs into various body fluids, including the blood and urine (48). In the current study, EVs were successfully isolated from the serum, which was consistent with previous studies $(49,50)$. EVs contain a wide range of DNA, mRNA, proteins, miRNA, lncRNA, circular RNA and metabolites (51). EVs are involved in the development of GC through intercellular communication (16). Therefore, EVs are regarded as suitable non-invasive biomarkers for GC. Multiple methods to obtain EVs are available, including density-gradient centrifugation, sucrose cushion centrifugation, 
gel-permeation chromatography, affinity capture, microfluidic devices, synthetic polymer-based precipitation and membrane filtration (52). The current study compared the efficiency of EV extraction by ultracentrifugation and a commercial kit. The results demonstrated that EVs were successfully obtained by both methods, and that their characteristics were similar, as determined by TEM, surface marker proteins, size distribution and zeta potential. Compared with the that obtained using the commercial kit, the yield of EVs extracted by ultracentrifugation was higher; however, the purity was lower. In addition, ultracentrifugation is time-consuming, thus it is recommended that commercial kits are used to obtain EVs in future studies.

1ncRNA CCAT1 silencing inhibits PANC-1 and Aspc-1 PC cell proliferation and migration, and rescues cyclin D1 expression (32). Another study has revealed that 1ncRNA CCAT1 promotes the proliferation and migration of HCC cells in vitro by depressing the expression levels of let-7, targeting high mobility group AT-hook 2 and c-Myc (31). The development of GC is a complex process, starting with chronic gastritis, which subsequently progresses to atypical hyperplasia and finally GC. Alterations in the resulting EV lncRNAs of these processes that are secreted into the periphery may therefore serve as suitable biomarkers.

The present study assessed the potential clinical value of EV lncRNA CCAT1. The results revealed that EV lncRNA CCAT1 levels in patients with GC were significantly higher compared with those in healthy control subjects, as well as in patients with chronic gastritis and atypical hyperplasia. These results suggested that EV IncRNA CCAT1 may be involved in GC progression. It is hypothesized that during GC progression, abnormal cells release EVs containing lncRNA CCAT1, which enter the periphery; once EVs bind to target cells, their contents can be transferred to the cytoplasm through a variety of pathways, thus altering the physiological state of the receptor cells (53). Kalluri and LeBleu (54) have demonstrated that EVs downregulate the levels of major histocompatibility complex-II transcription factor regulatory factor $\mathrm{X}$ associated protein in dendritic cells, potentially promoting immune evasion by cancer cells. In addition, EVs activate macrophages and increase the levels of proinflammatory cytokines, which in turn promote the proliferation and migration of tumor cells, leading to the reconstruction of the tumor microenvironment (55). In addition, atypical hyperplasia is a key step for the progression from a healthy state to GC (56). The results of the present study revealed that the expression levels of lncRNA CCAT1 in atypical hyperplasia were higher compared with those in healthy control subjects, which further confirmed its role in GC progression. A previous study has demonstrated that IncRNAs exist stably in the blood, possibly to protect EVs from degradation (44). The results of the current study demonstrated that serum EV lncRNA CCAT1 was expressed at stable levels, and that there were no significant changes in the expression levels of lncRNA CCAT1 at different times, temperatures and conditions of repeated freezing and thawing, suggesting that serum EV lncRNA CCAT1 may be suitable as a biomarker of GC. The results of the present study also demonstrated that lncRNA CCAT1 was associated with various clinical parameters that may be involved in the invasion and metastasis of GC. The mechanism by which lncRNA CCAT1 exerts its functions may be activated by c-Myc or the upregulation of Bmi-1 to promotes the proliferation, migration and invasion of GC cells $(36,57,58)$.

In the present study, EV lncRNA CCAT1 levels were markedly higher in patients with stage III+IV GC compared with those with stage I+II GC. These results suggested that with GC development, the expression levels of lncRNA CCAT1 in the serum were increased; thus, IncRNA CCAT1 may be associated with GC progression. ROC curve analysis revealed that EV 1ncRNA CCAT1 had a markedly higher diagnostic value compared with that of CEA. An increasing body of evidence has demonstrated that a combination of multiple tumor markers may improve the accuracy of diagnosis. For examples, Zhao et al (59) have reported that the combination of lncRNA CCAT1 and lncRNA HOX transcript antisense RNA provides more effective screening for CRC compared with HOTAIR or CCAT1 alone. In addition, Li et al (60) have demonstrated that the diagnostic value of EV lnc-GNAQ-6:1 was higher compared with that of traditional tumor markers CEA, CA 19-9 and CA72-4, and that the combination of these markers may provide an effective screening method for GC. The current study combined lncRNA CCAT1 and CEA to evaluate the diagnostic value of this combination in GC, and reached similar conclusions; the markers were more effective in combination compared with the results obtained with each marker alone.

Previous studies have suggested that serum/plasma EV lncRNA is secreted from tumor tissues. Tong et al (61) have reported that the expression of lncRNA POU class 3 homeobox 3 is consistent in the tissues and serum of patients with esophageal squamous cell carcinoma. The results of the present study demonstrated that the expression levels of lncRNA CCAT1 in GC tissues were higher compared with those in adjacent non-cancerous tissues and were positively correlated with those in the serum. It was hypothesized that lncRNA CCAT1 may be secreted by cancer cells during GC progression. The online database exoRBase analysis revealed that EV lncRNA CCAT1 levels were upregulated in hepatocellular carcinoma and pancreatic adenocarcinoma compared those in healthy subjects. However, there was a lack of data from patients with GC. Therefore, the current study may provide novel information for the GC diagnosis.

A previous study has demonstrated that lncRNAs are associated with the poor prognosis of several types of cancer including lung adenocarcinoma, hepatocellular carcinoma and melanoma (62). In the present study, patients with high expression levels of IncRNA CCAT1 presented with a poor prognosis. Similarly, a study by Ozawa et al (63) has determined that high lncRNA CCAT1 expression levels are significantly associated with low remission-free survival and OS rates in patients with CRC. Taken together, these results suggested lncRNA CCAT1 may be used as a biomarker to diagnose and determine the prognosis of patients with GC. However, there were limitations to the present study, including its retrospective nature and a relatively small sample size. Further studies with a larger sample size that aim to determine the underlying mechanism of EV IncRNA CCAT1 in GC progression are therefore required.

In conclusion, the results of the current study demonstrated that the levels of serum EV lncRNA CCAT1 were significantly upregulated in patients with GC compared with those in healthy subjects and patients with other illnesses, demonstrating the 
diagnostic value of this IncRNA. In addition, the levels of EV lncRNA CCAT1 were associated with the depth of invasion and metastasis. IncRNA CCAT1 existed stably in serum EVs and may be derived from GC cells. These results provide a foundation for further analysis of the clinical value of EV lncRNA CCAT1 in the diagnosis and prognosis of patients with GC.

\section{Acknowledgements}

The authors would like to thank Professor Dong Sun (Department of General Surgery, Qilu Hospital of Shandong University, Jinan, China) for their technical assistance.

\section{Funding}

This study was supported by The National Natural Science Foundation of China (grant nos. 82072358, 81972005 and 81572070), The Natural Science Foundation of Shandong Province (grant no. ZR2017MH044), the Key Research and Development Project of Shandong Province (grant nos. 2016GSF201122 and 2016GSF201124), the Science and Technology Development Project in Jinan (grant no. 201805084) and the Latitudinal Research Project of Shandong University (grant no. 6010119015).

\section{Availability of data and materials}

The datasets used and/or analyzed during the current study are available from the corresponding author on reasonable request.

\section{Authors' contributions}

YZ conceived the study, analyzed the data and reviewed the manuscript. KX designed the methodology, performed the analysis, prepared the original draft and was responsible for the project administration. DW was responsible for the bioinformatics and part of the statistical analysis. KX, ZD and DW extracted and identified EVs. WC, ZS and CY performed validation experiments, revised the manuscript and were involved in all the stages of the study. ZD and ML provided the information of patients and collected tissue samples of patients. JD, ZS collected blood samples of patients. KX prepared the graphs and figures. YZ supervised the study. ML and JD interpreted the data and participated in the writing of the manuscript. KX, ZD and DW confirm the authenticity of all the raw data. All authors read and approved the final version of the manuscript.

\section{Ethics approval and consent to participate}

This study was approved by Medical Ethics Committee of Qilu Hospital of Shandong University (approval no. KYLL-2015-097). All samples were anonymized during the analysis. All participants provided signed informed consent.

\section{Patient consent for publication}

Not applicable.

\section{Competing interests}

The authors declare that they have no competing interests.

\section{References}

1. Bray F, Ferlay J, Soerjomataram I, Siegel RL, Torre LA and Jemal A: Global cancer statistics 2018: GLOBOCAN estimates of incidence and mortality worldwide for 36 cancers in 185 countries. CA Cancer J Clin 68: 394-424, 2018.

2. Torre LA, Bray F, Siegel RL, Ferlay J, Lortet-Tieulent J and Jemal A: Global cancer statistics, 2012. CA Cancer J Clin 65: 87-108, 2015.

3. Smyth EC, Nilsson M, Grabsch HI, van Grieken NC and Lordick F: Gastric cancer. Lancet 396: 635-648, 2020.

4. Fu M, Gu J, Jiang P, Qian H, Xu W and Zhang X: Exosomes in gastric cancer: Roles, mechanisms, and applications. Mol Cancer 18: 41, 2019.

5. Dong Z, Sun X, Xu J, Han X, Xing Z, Wang D, Ge J, Meng L and $\mathrm{Xu} \mathrm{X}$ : Serum membrane type 1-matrix metalloproteinase (MT1-MMP) mRNA protected by exosomes as a potential biomarker for gastric cancer. Med Sci Monit 25: 7770-7783, 2019.

6. Wang J, Liu Y, Sun W, Zhang Q, Gu T and Li G: Plasma exosomes as novel biomarker for the early diagnosis of gastric cancer. Cancer Biomark 21: 805-812, 2018.

7. Hernández A, Arab JP, Reyes D, Lapitz A, Moshage H, Bañales JM and Arrese M: Extracellular vesicles in NAFLD/ALD: From pathobiology to therapy. Cells 9: 817, 2020.

8. Martinez RM, Hauser R, Liang L, Mansur A, Adir M, Dioni L, Racowsky C, Bollati V, Baccarelli AA and Machtinger R: Urinary concentrations of phenols and phthalate metabolites reflect extracellular vesicle microRNA expression in follicular fluid. Environ Int 123: 20-28, 2019.

9. Jun JH, Kim JY, Choi JH, Lim JY, Kim K and Kim GJ: Exosomes from placenta-derived mesenchymal stem cells are involved in liver regeneration in hepatic failure induced by bile duct ligation. Stem Cells Int 2020: 5485738, 2020.

10. Gholami L, Nooshabadi VT, Shahabi S, Jazayeri M, Tarzemany R, Afsartala Z and Khorsandi K: Extracellular vesicles in bone and periodontal regeneration: Current and potential therapeutic applications. Cell Biosci 11: 16, 2021.

11. Samanta S, Rajasingh S, Drosos N, Zhou Z, Dawn B and Rajasingh J: Exosomes: new molecular targets of diseases. Acta Pharmacol Sin 39: 501-513, 2018.

12. Maacha S, Bhat AA, Jimenez L, Raza A, Haris M, Uddin S and Grivel JC: Extracellular vesicles-mediated intercellular communication: Roles in the tumor microenvironment and anti-cancer drug resistance. Mol Cancer 18: 55, 2019.

13. Abd Elmageed ZY, Yang Y, Thomas R, Ranjan M, Mondal D, Moroz K, Fang Z, Rezk BM, Moparty K, Sikka SC, et al: Neoplastic Reprogramming of Patient-Derived Adipose Stem Cells by Prostate Cancer Cell-Associated Exosomes. Stem Cells 32: 983-997, 2014.

14. Zhang $\mathrm{X}$, Shi $\mathrm{H}$, Yuan $\mathrm{X}$, Jiang $\mathrm{P}$, Qian $\mathrm{H}$ and $\mathrm{Xu} \mathrm{W}$ : Tumor-derived exosomes induce N2 polarization of neutrophils to promote gastric cancer cell migration. Mol Cancer 17: 146, 2018.

15. Mulcahy LA, Pink RC and Carter DR: Routes and mechanisms of extracellular vesicle uptake. J Extracell Vesicles 3: 24641, 2014.

16. Huang T, Song C, Zheng L, Xia L, Li Y and Zhou Y: The roles of extracellular vesicles in gastric cancer development, microenvironment, anti-cancer drug resistance, and therapy. Mol Cancer 18: 62, 2019.

17. Xu R, Rai A, Chen M, Suwakulsiri W, Greening DW and Simpson RJ: Extracellular vesicles in cancer-implications for future improvements in cancer care. Nat Rev Clin Oncol 15: 617-638, 2018.

18. Lin MT, Song HJ and Ding XY: Long non-coding RNAs involved in metastasis of gastric cancer. World J Gastroenterol 24: 3724-3737, 2018.

19. Jiang S, Cheng SJ, Ren LC, Wang Q, Kang YJ, Ding Y, Hou M, Yang XX, Lin Y, Liang N and Gao G: An expanded landscape of human long noncoding RNA. Nucleic Acids Res 47: 7842-7856, 2019.

20. Zhou X, Chen H, Zhu L, Hao B, Zhang W, Hua J, Gu H, Jin W and Zhang G: Helicobacter pylori infection related long noncoding RNA (lncRNA) AF147447 inhibits gastric cancer proliferation and invasion by targeting MUC2 and up-regulating miR-34c. Oncotarget 7: 82770-82782, 2016.

21. Zhuo W, Liu Y, Li S, Guo D, Sun Q, Jin J, Rao X, Li M, Sun M, Jiang M, et al: Long noncoding RNA GMAN, up-regulated in gastric cancer tissues, is associated with metastasis in patients and promotes translation of ephrin A1 by competitively binding GMAN-AS. Gastroenterology 156: 676-691.e11, 2019. 
22. Yang L, Peng X, Li Y, Zhang X, Ma Y, Wu C, Fan Q, Wei S, Li H and Liu J: Long non-coding RNA HOTAIR promotes exosome secretion by regulating RAB35 and SNAP23 in hepatocellular carcinoma. Mol Cancer 18: 78, 2019.

23. Li Q, Shao Y, Zhang X, Zheng T, Miao M, Qin L, Wang B, Ye G, Xiao B and Guo J: Plasma long noncoding RNA protected by exosomes as a potential stable biomarker for gastric cancer. Tumour Biol 36: 2007-2012, 2015.

24. Lin LY, Yang L, Zeng Q, Wang L, Chen ML, Zhao ZH, Ye GD, Luo QC, Lv PY, Guo QW, et al: Tumor-originated exosomal lncUEGC1 as a circulating biomarker for early-stage gastric cancer. Mol Cancer 17: 84, 2018.

25. Zhao R, Zhang Y, Zhang X, Yang Y, Zheng X, Li X, Liu Y and Zhang Y: Exosomal long noncoding RNA HOTTIP as potential novel diagnostic and prognostic biomarker test for gastric cancer. Mol Cancer 17: 68, 2018

26. Arunkumar G, Murugan AK, Prasanna Srinivasa Rao H, Subbiah S, Rajaraman R and Munirajan AK: Long non-coding RNA CCAT1 is overexpressed in oral squamous cell carcinomas and predicts poor prognosis. Biomed Rep 6: 455-462, 2017.

27. Mizrahi I, Mazeh H, Grinbaum R, Beglaibter N, Wilschanski M, Pavlov V, Adileh M, Stojadinovic A, Avital I, Gure AO, et al: Colon cancer associated transcript-1 (CCAT1) expression in adenocarcinoma of the stomach. J Cancer 6: 105-110, 2015

28. Chen S, Liu Y, Wang Y and Xue Z: LncRNA CCAT1 promotes colorectal cancer tumorigenesis via a miR-181b-5p/TUSC3 axis. Onco Targets Ther 12: 9215-9225, 2019.

29. Deng L, Yang SB, Xu FF and Zhang JH: Long noncoding RNA CCAT1 promotes hepatocellular carcinoma progression by functioning as let-7 sponge. J Exp Clin Cancer Res 34: 18, 2015

30. Zhang C, Wang W, Lin J, Xiao J and Tian Y: IncRNA CCAT1 promotes bladder cancer cell proliferation, migration and invasion. Int Braz J Urol 45: 549-559, 2019.

31. Xin Y, Li Z, Shen J, Chan MT and Wu WK: CCAT1: A pivotal oncogenic long non-coding RNA in human cancers. Cell Prolif 49: 255-260, 2016.

32. Liu Z, Chen Q and Hann SS: The functions and oncogenic roles of CCAT1 in human cancer. Biomed Pharmacother 115: 108943 , 2019.

33. Shen H, Wang L, Xiong J, Ren C, Gao C, Ding W, Zhu D, Ma D and Wang H: Long non-coding RNA CCAT1 promotes cervical cancer cell proliferation and invasion by regulating the miR-181a-5p/MMP14 axis. Cell Cycle 18: 1110-1121, 2019.

34. You Z, Liu C, Wang C, Ling Z, Wang Y, Wang Y, Zhang M, Chen S, Xu B, Guan H and Chen M: LncRNA CCAT1 promotes prostate cancer cell proliferation by interacting with DDX5 and MIR-28-5P. Mol Cancer Ther 18: 2469-2479, 2019.

35. Abedini P, Fattahi A, Agah S, Talebi A, Beygi AH, Amini SM, Mirzaei A and Akbari A: Expression analysis of circulating plasma long noncoding RNAs in colorectal cancer: The relevance of lncRNAs ATB and CCAT1 as potential clinical hallmarks. J Cell Physiol 234: 22028-22033, 2019.

36. Li Y, Zhu G, Ma Y and Qu H: IncRNA CCAT1 contributes to the growth and invasion of gastric cancer via targeting miR-219-1. J Cell Biochem 120: 19457-19468, 2019.

37. Xie Y, Dang W, Zhang S, Yue W, Yang L, Zhai X, Yan Q and $\mathrm{Lu} \mathrm{J}$ : The role of exosomal noncoding RNAs in cancer. Mol Cancer 18: 37, 2019.

38. Barbagallo C, Brex D, Caponnetto A, Cirnigliaro M, Scalia M, Magnano A, Caltabiano R, Barbagallo D, Biondi A, Cappellani A, et al: LncRNA UCA1, upregulated in CRC biopsies and downregulated in serum exosomes, controls mRNA expression by RNA-RNA interactions. Mol Ther Nucleic Acids 12: 229-241, 2018

39. Galamb O, Barták BK, Kalmár A, Nagy ZB, Szigeti KA Tulassay Z, Igaz P and Molnár B: Diagnostic and prognostic potential of tissue and circulating long non-coding RNAs in colorectal tumors. World J Gastroenterol 25: 5026-5048, 2019.

40. Amin MB, Greene FL, Edge SB, Compton CC, Gershenwald JE, Brookland RK, Meyer L, Gress DM, Byrd DR and Winchester DP The eighth edition AJCC cancer staging manual: Continuing to build a bridge from a population-based to a more 'personalized' approach to cancer staging. CA Cancer J Clin 67: 93-99, 2017.

41. Zhang J, Arends RH, Kubiak RJ, Roskos LK, Liang M, Lee N, Chen CC and Yang $\mathrm{H}$ : A new method for identification of outliers in immunogenicity assay cut point data. J Immunol Methods 484-485: 112817, 2020.

42. Livak KJ and Schmittgen TD: Analysis of relative gene expression data using real-time quantitative PCR and the 2(-Delta Delta $\mathrm{C}(\mathrm{T})$ ) method. Methods 25: 402-408, 2001
43. Li S, Li Y, Chen B, Zhao J, Yu S, Tang Y, Zheng Q, Li Y, Wang P, He X and Huang S: ExoRBase: A database of circRNA, lncRNA and mRNA in human blood exosomes. Nucleic Acids Res 46 (D1): D106-D112, 2018.

44. Arita T, Ichikawa D, Konishi H, Komatsu S, Shiozaki A, Shoda K, Kawaguchi T, Hirajima S, Nagata H, Kubota T, et al: Circulating long non-coding RNAs in plasma of patients with gastric cancer. Anticancer Res 33: 3185-3193, 2013.

45. Zhong G, Wang K, Li J, Xiao S, Wei W and Liu J: Determination of serum exosomal H19 as a noninvasive biomarker for breast cancer diagnosis. Onco Targets Ther 13: 2563-2571, 2020.

46. Li N, Li J, Mi Q, Xie Y, Li P, Wang L, Binang H, Wang Q, Wang Y, Chen Y, et al: Long non-coding RNA ADAMTS9-AS1 suppresses colorectal cancer by inhibiting the Wnt/ $\beta$-catenin signalling pathway and is a potential diagnostic biomarker. J Cell Mol Med 24: 11318-11329, 2020.

47. Wang J, Pu J, Zhang Y, Yao T, Luo Z, Li W, Xu G, Liu J, Wei W and Deng Y: Exosome-transmitted long non-coding RNA SENP3-EIF4A1 suppresses the progression of hepatocellular carcinoma. Aging (Albany NY) 12: 11550-11567, 2020.

48. Taylor DD and Gercel-Taylor C: MicroRNA signatures of tumor-derived exosomes as diagnostic biomarkers of ovarian cancer. Gynecol Oncol 110: 13-21, 2008

49. Scavo MP, Cigliano A, Depalo N, Fanizza E, Bianco MG, Denora N, Laquintana V, Curri ML, Lorusso D, Lotesoriere C, et al: Frizzled-10 extracellular vesicles plasma concentration is associated with tumoral progression in patients with colorectal and gastric cancer. J Oncol 2019: 2715968, 2019.

50. Chung KY, Quek JM, Neo SH and Too HP: Polymer-based precipitation of extracellular vesicular miRNAs from serum improve gastric cancer miRNA biomarker performance. J Mol Diagn 22: 610-618, 2020.

51. Pegtel DM and Gould SJ: Exosomes. Annu Rev Biochem 88: 487-514, 2019.

52. Xu R, Greening DW, Zhu HJ, Takahashi N and Simpson RJ: Extracellular vesicle isolation and characterization: Toward clinical application. J Clin Invest 126: 1152-1162, 2016.

53. Tkach $\mathrm{M}$ and Théry $\mathrm{C}$ : Communication by extracellular vesicles: Where we are and where we need to go? Cell 164: 1226-1232, 2016

54. Kalluri R and LeBleu VS: The biology, function, and biomedical applications of exosomes. Science 367: eaau6977, 2020.

55. Wu L, Zhang X, Zhang B, Shi H, Yuan X, Sun Y, Pan Z, Qian H and $\mathrm{Xu} \mathrm{W}$ : Exosomes derived from gastric cancer cells activate $\mathrm{NF}-\kappa \mathrm{B}$ pathway in macrophages to promote cancer progression. Tumour Biol 37: 12169-12180, 2016

56. Kinoshita H, Hayakawa Y and Koike K: Metaplasia in the stomach-precursor of gastric cancer? Int J Mol Sci 18: 2063, 2017.

57. Yang F, Xue X, Bi J, Zheng L, Zhi K, Gu Y and Fang G: Long noncoding RNA CCAT1, which could be activated by c-Myc, promotes the progression of gastric carcinoma. J Cancer Res Clin Oncol 139: 437-445, 2013.

58. Li N, Jiang K, Fang LP, Yao LL and Yu Z: Knockdown of long noncoding RNA CCAT1 inhibits cell growth, invasion and peritoneal metastasis via downregulation of Bmi-1 in gastric cancer. Neoplasma 65: 736-744, 2018.

59. Zhao W, Song M, Zhang J, Kuerban M and Wang H: Combined identification of long non-coding RNA CCAT1 and HOTAIR in serum as an effective screening for colorectal carcinoma. Int J Clin Exp Pathol 8: 14131-14140, 2015.

60. Li S, Zhang M, Zhang H, Hu K, Cai C, Wang J, Shi L, $\mathrm{Ma} \mathrm{P}, \mathrm{Xu} \mathrm{Y}$ and Zheng P: Exosomal long noncoding RNA lnc-GNAQ-6:1 may serve as a diagnostic marker for gastric cancer. Clin Chim Acta 501: 252-257, 2020.

61. Tong YS, Wang XW,Zhou XL, Liu ZH, Yang TX, Shi WH, Xie HW, Lv J, Wu QQ and Cao XF: Identification of the long non-coding RNA POU3F3 in plasma as a novel biomarker for diagnosis of esophageal squamous cell carcinoma. Mol Cancer 14: 3, 2015 .

62. Zhang Z, Xie H, Liang D, Huang L, Liang F, Qi Q and Yang X Long non-coding RNA CCAT1 as a diagnostic and prognostic molecular marker in various cancers: A meta-analysis Oncotarget 9: 23695-23703, 2018.

63. Ozawa T, Matsuyama T, Toiyama Y, Takahashi N, Ishikawa T, Uetake H, Yamada Y, Kusunoki M, Calin G and Goel A: CCAT1 and CCAT2 long noncoding RNAs, located within the 8q.24.21 'gene desert', serve as important prognostic biomarkers in colorectal cancer. Ann Oncol 28: 1882-1888, 2017.

This work is licensed under a Creative Commons Attribution-NonCommercial-NoDerivatives 4.0 International (CC BY-NC-ND 4.0) License. 